\title{
Histological Observation on Adult Gonads from Meiogynogentic Olive Flounder Paralichthys olivaceus
}

\author{
Deyou Ma ${ }^{1,3}$, Shenda Weng ${ }^{2}$, Peng Sun ${ }^{5}$ Jun $\mathrm{Li}^{2,4}$, Peijun Zhang ${ }^{2}$ and Feng You ${ }^{2,4 *}$ \\ ${ }^{1}$ Key Laboratory of Mariculture \& Stock Enhancement in North China, Ministry of Agriculture, Dalian Ocean University, \\ Dalian-116023, China \\ ${ }^{2}$ Key Laboratory of Experimental Marine Biology, Institute of Oceanology, Chinese Academy of Sciences, Qingdao-266071, \\ China \\ ${ }^{3}$ Key laboratory of Fish Applied Biology and Aquaculture in North China, Liaoning Province, Dalian Ocean University, \\ Dalian-116023, China \\ ${ }^{4}$ Laboratory for Marine Biology and Biotechnology, Qingdao National Laboratory for Marine Science and Technology, \\ Qingdao-266071, China \\ ${ }^{5}$ Key Laboratory of East China Sea and Oceanic Fishery Resources Exploitation, Ministry of Agriculture, East China Sea \\ Fisheries Research Institute, Chinese Academy of Fishery Sciences, Shanghai-200090, China \\ *For correspondence: youfeng@qdio.ac.cn
}

\begin{abstract}
Gynogenesis is a common method to manipulate chromosomes of aquaculture animals with sex dimorphism. The adverse effects on gonad development can be identified through morphology and histology. The goal of this study was to examine the gonadal development of twenty-three meiogynogenetic olive flounder Paralichthys olivaceus samples of two-years age using histological and immunohistochemical methods. We found that ovaries of nine individuals developed normally, while those of fourteen fish exhibited some distinct malformation, including a pair of asymmetrically developed lobes (divided into only one lobe and one slowly developed lobe) and a pair of slowly developed lobes. The remaining two had unidentified gonads filled with connective tissue, indicating that gynogenesis induced by cold shock exerts adverse effect on this species. However, all meiogynogenetic ovaries developed to phase II, similar to the corresponding control. Furthermore, compared to the female references, the same level of P450arom positive responses was received in the aforementioned ovaries, and they were localized in the follicles and oocyte cytoplasms. The above-described results elucidated that meiogynogenetic P. olivaceus diploids are equipped with reproductive potential to undergo normal ovarian development and ovulation, suggesting they could be cultivated as broodstock to produce inbred lines for increase in flounder yields and enhancement of farmers' interests. (C) 2018 Friends Science Publishers
\end{abstract}

Keywords: Paralichthys olivaceus; Meiogynogenesis; Gonad development; Histological characteristics; P450arom

\section{Introduction}

Gynogenesis, a chromosome manipulation technique, is divided into two types according to methods of chromosomal diploidization: chromosomes of meiogynogenetic diploids (heterozygous) were reduplicated by suppressing the second polar body release from eggs fertilized with inactivated sperms, and thus mitogynogenetic diploids (homozygous) were chromosomally doubled by inhibiting the first egg cleavage (Arai, 2001). Gynogenesis is easily to be induced in fish and has been confirmed as an effective method to rapidly produce single-sex offspring in many freshwater species, such as zebrafish Danio rerio (Streisinger et al., 1981), medaka Oryzias latipes, common carp Cyprinus carpio L., Nile tilapia Oreochromis niloticus L., brook trout Salvelinus fontinalis, paddlefish Polyodon spathula, and bagrid catfish Pseudobagrus ussuriensis (Pan et al., 2017). In recent years, a number of studies on gynogenetic induction of marine fishes have been reported, such as red sea bream Pagrus major, turbot Scophthalmus maximus, European sea bass Dicentrarchus labrax, barfin flounder Verasper moseri, half-smooth tongue sole Cynoglossus semilaevis, Atlantic cod Gadus morhua L., olive flounder Paralichthys olivaceus, and yellow drum Nibea albiflora (Chen et al., 2017). On basis of the aforementioned induction results, the survival rate of mitogynogenetic fry is commonly much lower than that of the meiogynogenetic, so raising the latter is more desirable and of practical values.

Supposing that mass of viable meiogynogenetic diploids had been cultivated to be sexually mature and spawn naturally, they could act as broodstock to produce

To cite this paper: Ma, D., S. Weng, P. Sun, J. Li, P. Zhang and F. You, 2018. Histological observation on adult gonads from meiogynogentic olive flounder Paralichthys olivaceus. Int. J. Agric. Biol., 20: 689-694 
clones of females with hormonally sex-reversed male sibs (Komen and Thorgaard, 2007). The gonad development of meiogynogenetic stocks should be checked first before they are used for breeding. There were some studies focused on gonad development of gynogenetic fishes. In cyprinid loach Misgurnus anguillicaudatus (Suzuki et al., 1985), common carp Cyprinus carpio (Komen et al., 1992), Thai walking catfish Clarias macrocephalus (Na-Nakorn, 1995), sea bass Dicentrarchus labrax (Felip et al., 2002), turbot Scophthalmus maximus (Cal et al., 2006) and Atlantic cod Gadus morhua (Whitehead et al., 2012), gonads of their gynogenetic diploids showed no obvious differences compared with that of control in term of appearance and histological features. However, abnormally developed ovaries filled with a mass of immature oocytes were observed in fresh gynogenetics of coho salmon Oncorhynchus kisutch (Piferrer et al., 1994), and honmoroko Gnathopogon caerulescens (Fujioka, 1998). Regarding olive flounder $P$. olivaceus, although gonads of a few meiogynogenetic diploids were found to grow deformed in morphology (Tabata et al., 1986), but rare studies on their gonadal development using histological methods were reported to date, and consequently further investigation is required. During the ovarian development process, cytochrome $\mathrm{P} 450$ aromatase (P450arom in abbreviation) has been confirmed as the key enzyme to regulate estrogen conversion from androgen directly in various animals, including fishes (Kishida and Callard, 2001; Devlin and Nagahama, 2002), and then affect the developing status of oocytes. The high expression levels of P450arom encoding gene cyp19al are indispensable for ovarian differentiation in $P$. olivaceus. P450arom was chosen as a sensitive biomarker to label the developmental status of ovaries in gynogenetic fish.

$P$. olivaceus, mainly distributed in the coastal regions of China, Korea and Japan, has become an economically important marine fish species in North and even South China. Just as many other flatfishes present sexually dimorphic growth, females of flounder grow faster and reach a much larger size than males (Yamamoto, 1999). Producing all-female stocks is considered to be an efficient approach to significantly increase output of this cultured fish. In the present study, we investigated the gonad morphologies and histological development situation of adult (two-year age) meiogynogenetic flounders, and furthermore detected the developmental status of those gonads through immunohistological locating of P450arom. The results would be of great value to determine whether olive flounders induced by meiogynogenesis are suitable to be cultivated as broodstock for yielding all female progenies with cross of sex reversed males.

\section{Materials and Methods}

\section{Ethics Statement}

Experiments on flounder were performed on basis of the regulations of local and central government. All experiments were approved by the Institutional Animal Care and Use Committee of the Institute of Oceanology, Chinese Academy of Sciences.

\section{Meiogynogenetic Induction}

Eggs were artificially extruded from plump bellies of four female parent flounders, and fertilized with ultravioletirradiated heterogenous sperms (UV intensity, $150 \mathrm{~J} \mathrm{~cm}^{-2}$ ). Meiogenetic diploids were induced by cold shock at $0-1{ }^{\circ} \mathrm{C}$ for $45 \mathrm{~min}$ at $5 \mathrm{~min}$ after fertilization to suppress the release of the second polar body (You et al., 2001). Diploid control crossed by normal bisexual olive flounder gametes was used to check the gametic fertilization capability, meanwhile haploid control untreated by cold shock after fertilization was produced to assess the inactive effect of sperm by UV irradiation. The embryos and fries were cultivated in separate tanks at $15 \pm 1{ }^{\circ} \mathrm{C}$. The haploid control suffered the typical haploid syndrome (i.e. stubbed tails) could not hatch, while the meiogynogenetic diploid fry could undergo normal embryonic development, though some embryos died during hatching due to cold shock treatment. Subsequently, we identified their actual gynogenetic origin by microexamination of haploid control and a ploidy check using flow cytometry (data not shown). Finally, viable juvenile meiogynogens were cultured under indoor conditions (photoperiod: light $14 \mathrm{~h}$ and dark $10 \mathrm{~h}$, aeration, filtered seawater, water temperature range: $18 \sim 25^{\circ} \mathrm{C}$ ) and fed with commercial particle food twice a day until sampling.

\section{Histology}

Gonads were dissected from 23 randomly selected adult meiogynogens at 24-25 months of age (total body length: $31.4 \pm 2.1 \mathrm{~cm}$; body weight: $290.8 \pm 69.8 \mathrm{~g}$ ) after a mild anaesthetization using MS222 (SANDOZ, Switzerland), immediately fixed in Bouin's solution for $24 \mathrm{~h}$, later transferred to $70 \%$ ethanol, dehydrated by the increased ethanol gradients $(70 \% \sim 100 \%)$, and finally embedded in paraffin to finish the preparation of histological sections. The prepared samples were cut into slices with 5 7 $\mu \mathrm{m}$ thick, then dewaxed by the reduced ethanol gradients $(100 \% \sim 50 \%)$, cleaned up by distilled water, stained by hematoxylin-eosin (HE), and eventually examined microscopically after air drying. Ten control females with equal size (total body length: $32.1 \pm 1.7 \mathrm{~cm}$; body weight: 293.8 $\pm 20.3 \mathrm{~g})$ were cultivated under the same condition and treated by the same sampling process. The methods of handling control samples followed the above described. Each glass slide adhered five serial slices of one sample, respectively. One sample had three replicates. The gonad developmental phases were judged by evaluating the development of various gamete cells (such as size and ratio) comprehensively under optical microscope. 


\section{P450arom Immunohistochemistry}

In this study, we applied the streptavidin-biotin complex (SABC) method to locate P450arom in the aforementioned gonad slices. Antibody I was aromatase rabbit anti-human polyclonal antibody with dilution at $1: 150$ before use (Sigma, USA), and antibody II was biotin-goat anti-rabbit IgG (Boster, China). The gonad sections for immunochemistry were treated in advance: they were soaked by $4 \%$ polylysine for $5 \mathrm{~min}$ next to be dried at $37^{\circ} \mathrm{C}$ for $2 \mathrm{~h}$, then neutralized by $30 \% \mathrm{H}_{2} \mathrm{O}_{2}$ to clear endogenous peroxidase at room temperature for $10 \mathrm{~min}$, subsequently digested with $0.1 \%$ trpsin at $37^{\circ} \mathrm{C}$ for $30 \mathrm{~min}$ (sections and trypsin were both preheated to $37^{\circ} \mathrm{C}$ ), rinsed thoroughly with PBS for $5 \mathrm{~min}$, and finally sealed by bovine serum albumin (BSA) at room temperature for $20 \mathrm{~min}$. The following procedures included incubation of sections with antibody I at $4^{\circ} \mathrm{C}$ for $40 \mathrm{~h}$, later with biotin-antibody II at room temperature for $30 \mathrm{~min}$ after cleaning with PBS for $5 \mathrm{~min}$, then with $\mathrm{SABC}$ agent at room temperature for 20 min, cleanout of sections using PBS four times with interval of $5 \mathrm{~min}$ and final coloration with diaminobenzidine (DAB). Afterwards, these sections were washed with distilled water and re-stained by haematoxylin for $3 \mathrm{~s}$. The colors of slices were separated apart by hydrochloric acid-ethanol. Negative controls, derived from ovarian slices of female control, were produced by dropwise adding double distilled water on sections instead of antibody II (biotin-goat anti-rabbit IgG) after incubation with antibody I. In the end, the colored slices were dehydrated and enclosed by neutral gum for optically microscopic analysis.

\section{Results}

The characteristics of the gonad samples were compared between the adult meiogynogenetic flounders and female control. The results are presented in the following text and figures.

\section{Gonad Morphology}

We inspected the shape of gonads dissected from 23 meiogynogenetic flounder samples of two-year age. The results in Fig. 1A and D indicated that nine individuals possessed normal ovaries with unambiguous characters of control olive flounder. They all had a pair of ovaries with left-right symmetric lobes, lying in the body cavities similar to a pair of triangular halberds, containing two corresponding oviducts that merged to one duct near the genital pore. However, the remaining 14 samples had abnormal gonads. They were classified into ovaries with two asymmetrical lobes, including only one lobe (Fig. $1 \mathrm{E})$ or one lobe obviously shorter than the other one (Fig. 1F), and two symmetrical but evidently short lobes (Fig. 1G), as well sex-undistinguishable gonads under distinct developmental retardation (Fig. 1H).
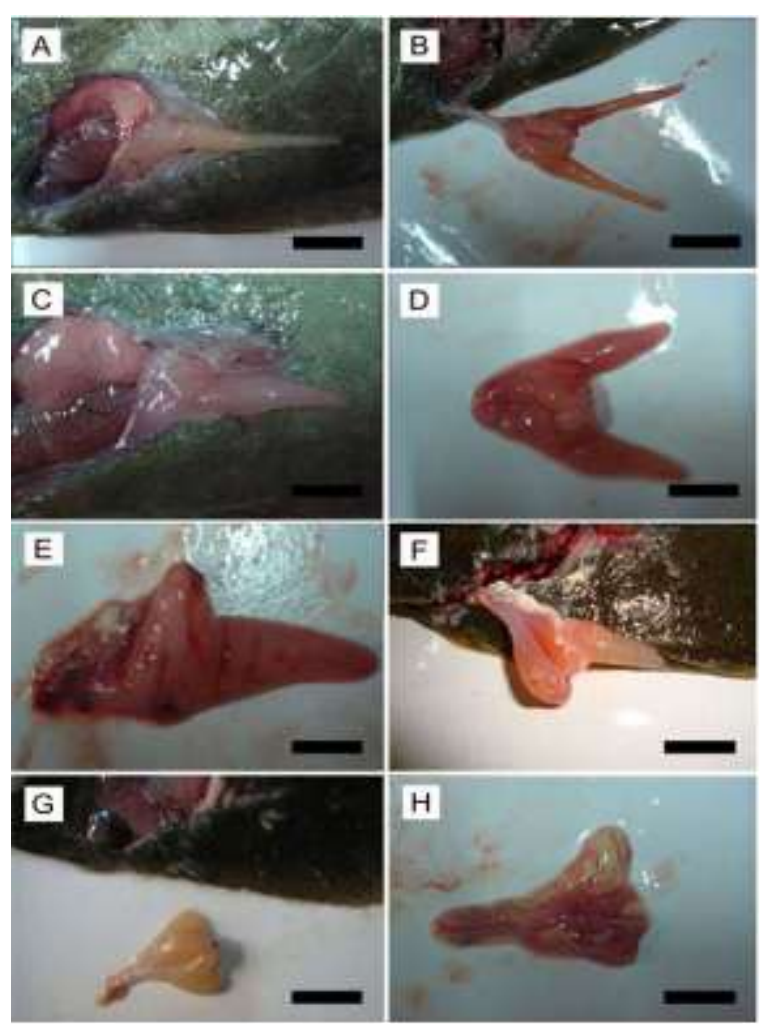

Fig. 1: External forms of ovaries separated from meiogynogenetic olive flounders: $(\mathrm{B}, \mathrm{D})$ morphologically normal ovaries dissected from corresponding samples (A, C); (E) the deformed meiogynogenetic ovaries possessing only one lobe; (F) one slowly developed lobes; (G) two slowly developed lobes; $(\mathrm{H})$ the slim and sexundistinguishable gonad. Scale bars $=1 \mathrm{~cm}$

Among 14 abnormal samples, there were nine with asymmetrically developed ovaries, three with symmetrically developed but slowly-developing ovaries, and two with slim and sex-undistinguishable gonads.

\section{Histological Structures}

The six-phase classification based on histological features of oocytes has been generally accepted in ovarian development of fish (Song and Wen, 2005). In this study, we determined that ovaries of female controls marched into phase II, according to the histological events in Fig. 2A and D, such as the increasing nucleus numbers, existence of oils and yolkvacuoles in occytes. The meiogynogenetic gonads were classified into ovaries with a pair lobes (Fig. 2E and F), only one lobe (Fig. 2G and H), one lobe slowly-developing (Fig. 2I and J), both lobes under development retardation (Fig. $2 \mathrm{~K}$ and $\mathrm{L}$ ), and their histological structures were nearly in line with the control to stay at phase II, exlusive of the slim and sexundistinguishable gonads filled with connective tissue and few oogonia (Fig. 2M and N). 


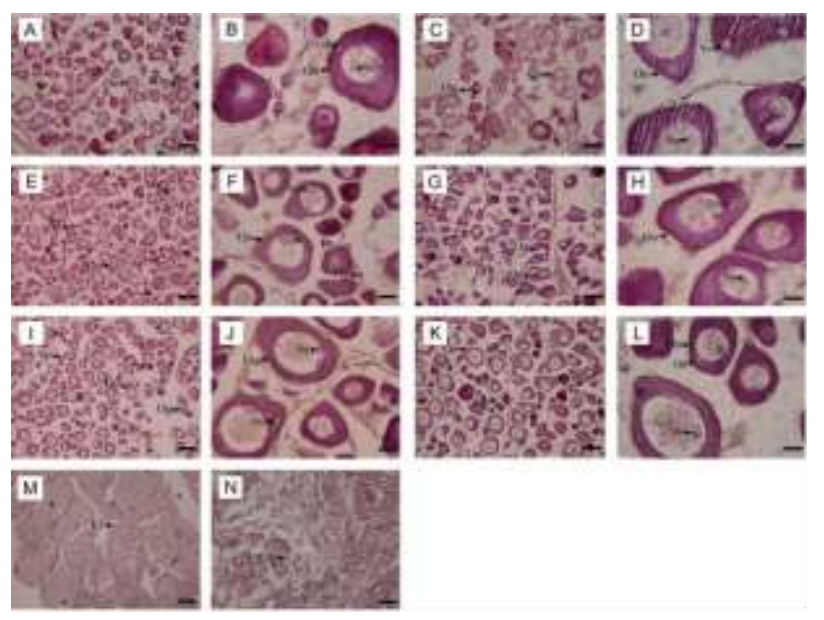

Fig. 2: Histological structures of gonad sections cut from the control and meiogynogenetic olive flounders by $\mathrm{HE}$ staining: (A, C) ovaries separated from two control female flounders; (E, G, I, K) meiogynogenetic fish samples possessing two normal, only one, one or two slowly developed lobes, respectively; (M) the slim and sexundistinguishable gonad. (B, D, F, H, J, L, N) 5 folds amplification of (A, C, E, G, I, K, M), respectively. Scale bars in (A, C, E, G, I, K) were $100 \mu \mathrm{m}$, and in (B, D, F, H, $\mathrm{J}, \mathrm{L}, \mathrm{N}) 20 \mu \mathrm{m}$. Abbreviations and symbols: Ct, connective tissue; $\mathrm{Nu}$, nucleus; Oo, oocyte; Og, oogonium; $\mathrm{Sp}$, spawning plank; Yv, yolk vacuole

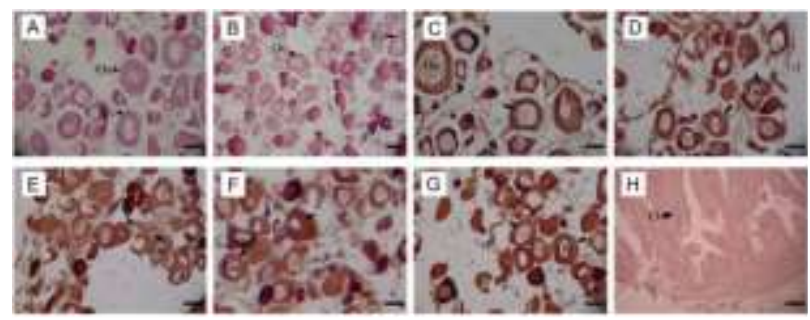

Fig. 3: Observation of P450arom localized in ovaries of the female control and meiogynogenetic samples by immunohistochemical staining: (A, B, H) P450arom negative reactions in ovaries of one control female, one meiogynogenetic flounder with two normal lobes, slim and sex-undistinguishable gonads $(\mathrm{H})$, respectively; $(\mathrm{C}, \mathrm{D}, \mathrm{E}$, $\mathrm{F}, \mathrm{G})$ positive responses in the corresponding control, meiogynogenetic ovaries with two normal, only one, one or two slowly developed lobes, respectively. Arrows indicates P450arom-positive locations. Scale bar $=100 \mu \mathrm{m}$. Abbreviations and symbols: $\mathrm{Ct}$, connective tissue; Fc, follicle; Oo, oocyte

\section{P450arom Location}

In the present study, we observed the gonad samples of these 23 adult meiogynogenetic flounder samples using P450arom immunohistochemistry for further study on their developmental conditions. The results indicated that cells of one meiogynogenetic sample with normal ovaries were stained lilac by hematoxylin, similar to the control acting as P450arom immuno-negative controls (Fig. 3A and B). Aromatase-positive cells were painted into deep brown on the clear background, and consequently they were easily discovered. The P450arom immuno-active signals were not only detected in the follicles and oocyte cytoplasms of control ovaries (Fig. 3C), but also meiogynogenetic ovaries having two normal lobes (Fig. 3D), only one lobe (Fig. 3E), and two lobes with one (Fig. 3F) or both short (Fig. 3G), whose reactive intensities among these ovaries were consistent except the thread-like and sex-undistinguishable gonad (Fig. 3H).

\section{Discussion}

According to prior studies on gonad development of female fish, most of them reach to the first sexual maturity 24 months post hatch and will soon undergo a reproductive cycle (Yamamoto, 1999; Felip et al., 2002), indicating that two years of age is a key time point to inquire gonad growth status for fish broodstock choice, especially artificially induced species. Our investigation revealed that the more than $90 \%(21 / 23)$ meiogynogenetic samples of two-year age were female after dissection except two indistinguishable gonads, indicating male heterogamety of olive flounder (Tabata, 1991). Similar results were as well reported in fishes belonging to Cyprinid (Nagy et al., 1978) and Salmonidae (Piferrer et al., 1994), of which all or almost gynogenetic females have been produced. However, a high ratio of males had ever been observed in gynogenetic $P$. olivaceus. Furthermore, the same confusion has been found in lots of other gynogenetically induced fish, such as zebrafish (Streisinger et al., 1981), carp species (Komen et al., 1992), honmoroko Gnathopogon caerulescens (Fujioka, 1998), and sea bass Dicentrarchus labrax (Felip et al., 2002; Francescon et al., 2005). Researchers considered that males of gynogenetic honmoroko could be masculinized females (Fujioka, 1998). In our study, it is estimated that the two meiogynogenetic samples carrying undeveloped gonads could suffer sex-reverse caused by high amient temperature during the key sex determination period (Yamamoto, 1999). Certainly, abundant samples are required for investigating the veritable sex ratio of meiogynogenetic groups of this important economic species.

It has been well-documented that cold shock has a negative impact on development of gonads of gynogenetically induced fish such as coho salmon (Piferrer et al., 1994) and common carp (Nagy et al., 1978). In the present work, four types of gonad malformation were found in over three-fifth of flounder samples for the first time, including ovaries possessing only one lobe, two lobes with one or both short, and thread-like gonads. 
The results indicated that the probability of gonad deformity was under high risk after cold shock induction in $P$. olivaceus and the harmful effect was definitely revealed in gonads of grown meiogynogenetic flounders. It is concluded that inbreeding effects or expression of deleterious recessive genes related to reproduction might be responsible for the gynogenetic gonad abnormality of various fish species (Purdom, 1983; Chourrout, 1984; Piferrer et al., 1994; Fujioka, 1998; Krisfalusi et al., 2000; Arai, 2001). The undifferentiated gonads full of connective tissues along with rarely seen primordial germ cells were likewise observed in gynogens of sea bass (Felip et al., 2002; Francescon et al., 2005) and honmoroko (Fujioka, 1998), speculating alterations in gonadal development of adult meiogynogenetic $P$. olivaceus under natural conditions. However, the mechanism on deviant growth of gynogenetic fish's gonads is still fascinating and needs to elucidated in multi-aspects, for example analysis among control, nornal and malformed gynogenetic gonads using "omics" technologies to explore massive genes and important pathways closely related to sex determation and gonad development of fish (Fan et al., 2014).

Even if the morphologically deficient gonads were found in the meiogynogenetic samples, their ovaries except thread-like undeveloped gonads all proceeded into phase II and displayed a synchronous development level when compared to control females in view of histological results. Similar cases were observed in the gynogenetic gonads of muskellunge (Rlnchard et al, 2002) and bagrid catfish (Park et al, 2007). The yolk vacuoles, landmark of early stage III ovaries, only emerged from one ovarian slice of control female flounders in Fig. 2A. Consequently this finding indicated that the development of these meiogynogenetic ovaries is slightly delayed in comparison with the corresponding control female samples. The similar performance was noticed in gynogenetic diploids of turbot (Cal et al., 2006) and Atlantic cod (Whitehead et al., 2012). We continued a higher-level inquiry into the development of those gynogenetic gonads via localizing P450 aromatase that has been proved to play a major role in ovarian development of multiple fish (Kishida and Callard, 2001; Kitano et al., 2002). Regarding P. olivaceus, the ovarian P450arom encoding gene cyp19a of this species was identified in relevance to reproductive traits (Wen et al., 2015), and suppression of this gene expression led to production reduction of estrogen and immediately block the ovarian development (Yamaguchi et al., 2007). The previous studies paid attention to the relation between cyp19a gene expression and gonadal development. In the present research, mammal antibody of P450arom was used to further check developmental status of gonad in lower vertebrates including fish by means of immunohistochemical localization, which has been validated available as the result of its high conservation. The positive P450arom responses were detected in ovarian follicles of these studied ovaries and their intensity was close to that of control flounder, which was similar with the locations in ovaries of yellowtail clownfish (Kobayashi et al., 2010). Comprehensively considering the above described results and the document of viable offsprings successfully produced by meiotic gynogenetic flounder broodstock (Jiang et al., 2017), it is reasonable to suggest that the mature meiogynogenetic ovaries of olive flounders could have the potencies of normal reproductive processes to produce sufficient estrogen, go through normal ovulation and spawn good eggs upon artificial treatment, even for these with malformed ovaries (Felip et al., 2002), as following of meiogynogenetic diploids induced from cyprinid loach Misgurnus anguillicaudatus (Suzuki et al., 1985), Thai walking catfish Clarias macrocephalus (Na-Nakorn, 1995), sea bass Dicentrarchus labrax (Francescon et al., 2005), and turbot Scophthalmus maximus (Cal et al., 2006).

\section{Conclusion}

In conclusion, according to the anatomic results it is authentic that cold shock exerts some harm on genetic materials of heterogeneously fertilized oocytes during gynogenetic induction of $P$. olivaceus, resulting in various degrees of shape deformity in gonads of adult fish. However, the histological and immunohistochemical examinations definitely indicate that the developmental phases of meiogynogenetic ovaries are fundamentally synchronized with that of corresponding control, implying that meiogynogenetic females of flounder could undergo normal ovarian development process, even gametogenesis and spawning.

\section{Acknowledgements}

This work was supported by the grants from the National Natural Science Foundation of China (Nos. 31772834 and 41276171), the National Flatfish Industry System Construction Programme of China (No. nycytx-50), the Scientific Research Project of Education Department of Liaoning Province (No. L201616) and the Doctor Initiative Research Program of Dalian Ocean Univerisity.

\section{References}

Arai, K., 2001. Genetic improvement of aquaculture finfish species by chromosome manipulation techniques in Japan. Aquaculture, 197: 205-228

Cal, R., S. Vidal, P. Martínez, B. Álvarez-Blázquez, C. Gómez and F Piferrer, 2006. Growth and gonadal development of gynogenetic diploid Scophthalmus maximus. J. Fish Biol., 68: 401-413

Chen, R., B. Lou, D. Xu, W. Zhan, Y. Takeuchi, F. Yang and F. Liu, 2017 Induction of meiotic gynogenesis in yellow drum (Nibea albiflora, Sciaenidae) using heterologous sperm and evidence for female homogametic sex determination. Aquaculture, 479: 667-674

Chourrout, D., 1984. Pressure-induced retention of second polar body and suprression of first cleavage in rainbow trout, production of alltriploids, all-tetraploids, heterozygous and homozygous diloid gynogenetics. Aquaculture, 36: 111-126 
Devlin, R.H. and Y. Nagahama, 2002. Sex determination and sex differentiation in fish, an overview of genetic, physiological, and environmental influences. Aquaculture, 208: 191-364

Fan, Z., F. You, L. Wang, S. Weng, Z. Wu, J. Hu, Y. Zou, X. Tan and P. Zhang, 2014. Gonadal transcriptome analysis of male and female olive flounder (Paralichthys olivaceus). Biomed Res. Int., 2014: 1-10

Felip, A., F. Piferrer, M. Carrillo and S. Zanuy, 2002. Growth, gonadal development and sex ratios of meiogynogenetic diploid sea bass. $J$. Fish Biol., 61: 347-359

Francescon, A., A. Barbaro, D. Bertotto, A. Libertini, F. Cepollaro, J. Richard, P. Belvedere and L. Colombo, 2005. Assessment of homozygosity and fertility in meiotic gynogens of the European sea bass (Dicentrarchus labrax L.). Aquaculture, 243: 93-102

Fujioka, Y., 1998. Survival, growth and sex ratios of gynogenetic diploid honmoroko. J. Fish Biol., 52: 430-442

Jiang, H.B., H.J. Liu, G.X. Wang, X.Y. Zhang, J. Bao and L. Jiang, 2017. Fast development of genetically uniform strains by gynogenesis in Japanese flounder (Paralichthys olivaceus). Aquac. Res., 48: 20322038

Kishida, M. and G.V. Callard, 2001. Distinct cytochrome P450 aromatase isoforms in zebrafish (Danio rerio) brain and ovary are differentially programmed and estrogen regulated during early development. Endocrinology, 142: 740-750

Kitano, T., K. Takamune, Y. Nagahama and S.I. Abe, 2002. Gonadal sex differention in Japanese flounder (Paralichthys olivaceus). Fish Sci., 68: 679-680

Kobayashi, Y., R. Horiguchi, S. Miura and M. Nakamura, 2010. Sex- and tissue-specific expression of $\mathrm{P} 450$ aromatase (cyp19ala) in the yellowtail clownfish, Amphiprion clarkii. Comp. Biochem. Phys. A, 155: 237-244

Komen, H. and G.H. Thorgaard, 2007. Androgenesis, gynogenesis and the production of clones in fishes, A review. Aquaculture, 269: 150-173

Komen, J., G.F. Wiegertjes, V.J.T. van Ginneken, E.H. Eding and C.J.J. Richter, 1992. Gynogenesis in common carp (Cyprinus carpio L.) III. The effects of inbreeding on gonadal development of heterozygous and homozygous gynogenetic offspring. Aquaculture, 104: $51-66$

Krisfalusi, M., P.A. Wheeler, G.H. Thorgaard and J.G. Cloud, 2000. Gonadal morphology of female diploid gynogenetic and triploid rainbow trout. J. Exp. Zool., 286: 505-512

Na-Nakorn, U., 1995. Comparison of cold and heat shocks to induce diploid gynogenesis in Thai walking catfish (Clarias macrocephalus) and performances of gynogens. Aquat. Living Resour., 8: 333-341

Nagy, A., K. Rajki, L. Horvárth and V. Csárnyi, 1978. Investigation on carp, Cyprinus carpio L. gynogenesis. J. Fish Biol., 13: 215-224

Pan, Z.J., C.K. Zhu, H. Wang, G.L. Chang, H.Y. Ding, X.G. Qiang and X.S. Yu, 2017. Induction of meiotic gynogenesis in bagrid catfish (Pseudobagrus ussuriensis) with homologous sperm and its confirmation for female homogamety. Aquac. Res., 48: 5659-5665
Park, S.Y., Y.K. Nam and I.C. Bang, 2007. Production of induced gynogenetic diploid bagrid catfish Leiocassis ussuriensis (Siluriformes) -II. Viability, early growth and sex ratio of gynogenetic diploidy. J. Aquacult., 20: 190-193

Piferrer, F., T. Benfey and E. Donaldson, 1994. Gonadal morphology of normal and sex-reversed triploid and gynogenetic diploid coho salmon (Oncorhynchus kisutch). J. Fish Biol., 45: 541-553

Purdom, C., 1983. Genetic engineering by the manipulation of chromosomes. Aquaculture, 33: 287-300

Rlnchard, J., M.A. Garcia-Abiado, K. Dabrowski, J. Ottobre and D. Schmidt, 2002. Induction of gynogenesis and gonad development in the muskellunge. J. Fish Biol., 60: 427-441

Song, H.X. and H.S. Wen, 2005. Histological studies on the ovarian development and its mechanism of regulation-controlling in cultured Japanese flounder Paralichthys olivaceus. T. Oceanol. Limnol., 4: $75-83$

Streisinger, G., C. Walker, N. Dower, D. Knauber and F. Singer, 1981. Production of clones of homozygous diploid zebra fish (Brachydanio rerio). Nature, 291: 293-296

Suzuki, R., T. Oshiro and T. Nakanishi, 1985. Survival, growth and fertility of gynogenetic diploids induced in the cyprinid loach, Misgurnus anguillicaudatus. Aquaculture, 48: 45-55

Tabata, K., 1991. Induction of gynogenetic dipploid males and presumption of sex determination mechanisms in the hirame Paralichthys olivaceus. Nippon Suisan Gakk., 57: 845-850

Tabata, K., S. Gorie and K. Nakamura, 1986. Studies on the gynogenesis in hirame Paralichthys olivaceus II. Induction of gynogenetic diploid in hirame Paralichthys olivaceus. Nippon Suisan Gakk., 52: 1901-1904

Wen, A., F. You, P. Sun, J. Li, D. Xu, Z. Wu, D. Ma, Y. Zou, X. Tan, Z. Fan and P. Zhang, 2015. Sexually dimorphic gene expression patterns during gonadal differentiation in olive flounder, Paralichthys olivaceus. Anim. Biol., 65: 193-207

Whitehead, J.A., T.J. Benfey and D.J. Martin-Robichaud, 2012. Ovarian development and sex ratio of gynogenetic Atlantic cod (Gadus morhua). Aquaculture, 324-325: 174-181

Yamaguchi, T., S. Yamaguchi, T. Hirai and T. Kitano, 2007. Folliclestimulating hormone signaling and Foxl2 are involved in transcriptional regulation of aromatase gene during gonadal sex differentiation in Japanese flounder, Paralichthys olivaceus. Biochem. Biophys. Res. Commun., 359: 935-940

Yamamoto, E., 1999. Studies on sex-manipulation and production of cloned populations in hirame, Paralichthys olivaceus (Temminck et Schlegel). Aquaculture, 173: 235-246

You, F., J. Liu, X.C. Wang, Y.L. Xu, R.D. Huang and P.J. Zhang, 2001 Study on embryonic development and early growth of triploid and gynogenetic diploid left-eyed flounder, Paralichthys olivaceus (T. et S.). Chin. J. Oceanol. Limnol., 19: 147-151

(Received 07 December 2017; Accepted 13 December 2017) 